\title{
Sources of Mathematics Self-Efficacy in Chinese Students: a Mixed-Method Study with Q-Sorting Procedure
}

\author{
Jie Gao ${ }^{1}$
}

Received: 5 October 2018 / Accepted: 3 May 2019 / Published online: 28 May 2019

(C) The Author(s) 2019

\begin{abstract}
Understanding the development of mathematics self-efficacy has important implications. Based on Bandura's four sources of self-efficacy, most previous studies mainly examined whether the sources predicted or correlated with mathematics self-efficacy, which might have oversimplified the underlying mechanism of self-efficacy development. This study conducted an in-depth investigation into the sources of mathematics self-efficacy of Chinese junior high school students. A mixed-method approach was adopted, which incorporated semi-structured interview with the "Q-sorting" procedure. Twelve Chinese students ( 6 boys and 6 girls, mean age 13.2 years) were purposely selected based on gender and their mathematics self-efficacy levels. Findings showed that students with either high or low self-efficacy not only experienced different degrees of exposure to the sources of self-efficacy but also held diverse viewpoints about the effects of each source on their mathematics self-efficacy. Girls recalled receiving more social persuasion and experiencing more anxiety than boys in mathematics learning. Yet, girls' perceptions of the influence of each source were not significantly different from boys'. In contrast to previous studies conducted in western contexts, this study demonstrates Chinese students' different viewpoints about the influence of effort, competition and face issue on their mathematics self-efficacy. Findings of this study shed light on the complex mechanism underlying the development of self-efficacy in mathematics learning.
\end{abstract}

Keywords Chinese students · Mathematics self-efficacy · Mixed-method · Q-sorting · Semi-structured interview

Electronic supplementary material The online version of this article (https://doi.org/10.1007/s10763-01909984-1) contains supplementary material, which is available to authorized users.

Jie Gao

J.Gao@shu.ac.uk

1 Department of Psychology, Sociology \& Politics, Sheffield Hallam University, Collegiate Crescent, Sheffield S10 2BQ, UK 


\section{Introduction}

As a crucial construct of Bandura's social cognitive theory (1997), self-efficacy refers to "beliefs in one's capabilities to organise and execute the courses of action required to produce given attainment" (Bandura, 1997, p. 3). Self-efficacy influences human functioning through cognitive, motivational, affective and selective processes. In educational contexts, a great many studies have substantiated the considerable effects of self-efficacy on students' academic motivation (e.g. Zimmerman, 2000), academic achievements (e.g. Prat-Sala \& Redford, 2012), learning strategies (e.g. Phan, 2011) and perseverance in face of academic setbacks (e.g. Caprara et al., 2008). Given such vigorous evidence, it is meaningful for educational researchers and practitioners to explore the mechanisms underlying the development of self-efficacy.

\section{Bandura's Four Sources of Self-Efficacy}

According to Bandura (1997), individuals develop and shape their self-efficacy by interpreting, weighing and integrating information from four sources, namely, mastery experience, vicarious learning, social persuasion and physiological and affective states.

Mastery experience refers to first-hand experience of success and failure. Empirical studies have generated substantial evidence of mastery experience being the most influential source of self-efficacy (Butz \& Usher, 2015; Joët, Usher, \& Bressoux, 2011). Yet, one must bear in mind that (a) it is not the mastery experience per se but rather the interpretation of the experience that shapes self-efficacy and (b) the ways in which students construe mastery experience are affected by both personal and contextual factors (Usher, 2009).

Meanwhile, students also gain efficacy-relevant information from vicarious learning which refers to learning from models about the requisite knowledge and skills to complete a task (Bandura, 1997). Modelling often facilitates observers' competence and therefore its instructive function promotes observers' self-efficacy. Yet, modelling also entails a comparative function, that is, students comparing themselves with the model when making self-appraisal (Chan \& Lam, 2008). Social comparative information serves as a double-edged sword to self-efficacy. On the one hand, it enables students to gain knowledge of their relative abilities, which may result in more accurate self-judgement of abilities. On the other hand, over-emphasis on comparison is likely to dampen motivation and lead to a decrease in self-efficacy (Chan \& Lam, 2008).

In addition to mastery and vicarious experiences, self-efficacy is also affected by social persuasion. It is not uncommon for students' self-efficacy to be influenced by evaluation from important others, such as parents, teachers and peers. Finally, individuals' physiological and affective states also affect self-efficacy through eliciting divergent interpretations of somatic and emotional arousals. Just as Bandura (1997) concluded, "efficacy beliefs are the product of cognitive processing of diverse sources of efficacy information conveyed enactively, vicariously, socially, and physiologically" (p. 115).

\section{Empirical Studies of the Sources of Self-Efficacy}

Based on Bandura's (1997) theory, a great many studies have examined the four sources of self-efficacy in educational contexts (see Usher \& Pajares, 2008 for a 
review). The most commonly adopted method in this field is survey research, which uses standardised measures to examine the relationship between the sources and selfefficacy. While survey research mainly assesses correlations, a few experimental/quasiexperimental studies have been conducted to investigate the effects of informational sources on students' self-efficacy by comparing experimental and control groups (e.g. Chan \& Lam, 2008). In addition to quantitative research, there are some qualitative studies which attempted to conduct more thorough investigations of how students cognitively process the information of efficacy sources, such as interview (e.g. Usher, 2009; Zeldin \& Pajares, 2000; Zeldin, Britner, \& Pajares, 2008), case study (e.g. Burnham, 2011) and thought-listing technique (e.g. Lent, Brown, Gover, \& Nijjer, 1996a).

Yet, very few studies managed to differentiate the following two issues, let alone to explore them respectively, that is, students not only experience different degrees of exposure to the sources of self-efficacy but also hold diverse viewpoints of the influence of each source on their self-efficacy. Nonetheless, evidence in favour of distinguishing these two issues is convincing when we examine individual and contextual factors which affect students' perceptions of self-efficacy sources, particularly with regard to gender, pre-existing self-efficacy level and cultural background.

Although gender difference has been widely explored, inconsistent findings were reported (see Usher \& Pajares, 2008 for review). In terms of mathematics, which was traditionally seen as a male-dominated subject, some studies found that boys reported more mastery experiences than girls (e.g. Joët et al., 2011) whereas girls acquired more efficacy-relevant information from social persuasion and vicarious experience (e.g. Lent, Lopez, Brown, \& Gore, 1996b; Lopez \& Lent, 1992). Similarly, in the subject of science which was also seen as male-dominated, Britner and Pajares (2006) found that boys reported more mastery experiences and lower level of anxiety than girls. Nonetheless, findings with regard to the influential power of the sources have sent a mixed message. Kiran and Sungur (2012) found that there was no significant gender difference in the relationship between the four hypothesised sources and science selfefficacy, even though girls did experience more anxiety than boys when engaged in science activities. In contrast, Usher and Pajares $(2006 \mathrm{a}, \mathrm{b})$ found that social persuasion predicted middle school girls' academic self-efficacy but not boys' (2006a) whereas physiological states predicted boys' self-efficacy but not girls' (2006b). In addition to aforementioned survey research, Zeldin and her colleagues interviewed both male and female professionals in science, mathematics and technology, reporting that social persuasions and vicarious experiences, particularly those from significant others, play a considerably vital role in the development of self-efficacy of females in maledominated arenas (Zeldin et al., 2008; Zeldin \& Pajares, 2000). Given these inconsistent findings, it is worthwhile to further explore gender difference in terms of the degree of exposure to sources of self-efficacy and the influential power of each source, respectively. By explicitly distinguishing these two issues, the present study sought to bring about new insights into the research of potential gender difference in selfefficacy development.

Bandura (1997) pointed out that pre-existing self-efficacy acts as a lens through which individuals construe new self-efficacy information. Usher's (2009) semistructured interview study with middle school students provided empirical evidence to support this argument. She found considerable difference in both the experiences and 
the interpretations of self-efficacy sources between students with different levels of mathematics self-efficacy. High self-efficacy students not only were exposed to more mastery experiences, more positive persuasion as well as fewer anxious/irritable arousals, but also were inclined to interpret the sources of self-efficacy in a more favourable manner in comparison to their low self-efficacy counterparts. However, other than Usher's (2009) study, there have not been adequate empirical studies explicitly investigating the difference between high and low self-efficacy students. The present study intends to address this gap and contribute to revealing how preexisting self-efficacy affects students' cognitive processing of new efficacy-related information.

In addition to the above internal factors, a variety of contextual factors also have been examined to account for individual difference in students' experiences and interpretations of self-efficacy sources (Usher \& Pajares, 2008), among which cultural background is an important one (Oettingen, 1995). Empirical evidence is in support of difference in sources of self-efficacy between students from individualistic and collectivistic cultural backgrounds. For example, Ahn, Usher, Butz, and Bong (2016b) found that socially conveyed sources of self-efficacy (i.e. vicarious experience and social persuasion) were construed and evaluated in different ways between students from the USA and South Asia. In a similar vein, Klassen (2004) found that South Asian immigrant students were significantly more susceptible to social comparative information than their Anglo Canadian non-immigrant counterparts. This is echoed by the finding in a quasi-experimental study of Chan and Lam (2008) who found that highlighting the comparative function of modelling significantly undermined Chinese students' self-efficacy. Apart from these findings, the number of studies that involved students of collectivistic cultural backgrounds is far from adequate (Usher \& Pajares, 2008). Therefore, in order to gain more insights into possible cultural variation in the sources of self-efficacy, empirical studies with samples of non-western cultural backgrounds are needed.

\section{Current Study}

Based on the discussion above, the present study proposes an in-depth investigation into Chinese junior high school students' experiences of the sources of self-efficacy as well as their perceptions of the influence of each source. The investigation aims to explore

- What experiences do Chinese students of different genders and self-efficacy levels have in terms of Bandura's four sources of self-efficacy in mathematics learning?

- How do Chinese students of different genders and self-efficacy levels perceive the influence of each of Bandura's self-efficacy sources on their mathematics selfefficacy?

The investigation adopts a mixed-method approach, that is, incorporating semistructured interview with the "Q-sorting" procedure (Stephenson, 1953), which has multiple methodological advantages in studying the sources of self-efficacy. According to Usher and Pajares (2008), existing quantitative research mainly focused on the degree to which each source predicts or correlates with mathematics self-efficacy, 
which may have oversimplified the complexity of self-efficacy development. By conducting the semi-structured interview, participants are allowed the opportunity to provide a more detailed and in-depth account of their perceptions of the sources of mathematics self-efficacy. Meanwhile, the "Q-sorting" procedure (more details in the "Method" section) provides a structure, with which participants could present their viewpoints in a more systematic and holistic manner. More importantly, "Q-sorting" enables participants to differentiate the effects of each source on their mathematics selfefficacy. By synthesising the findings of both qualitative and quantitative analysis, we could gain a more comprehensive understanding of how Chinese students perceive the sources of mathematic self-efficacy.

\section{Method}

\section{Participants}

Participants were recruited from a junior high school in a city in East China. Fiftythree students (34 boys and 19 girls, mean age 13.5 years) completed a questionnaire assessing their mathematics self-efficacy. Based on the result of mathematics self-efficacy level $(M=78.66 ; \mathrm{SD}=17.80 ; \mathrm{Mdn}=83.00)$ as well as the gender of students, 12 participants (6 boys and 6 girls) were purposely selected to take part in the semi-structured interview with the Q-sorting procedure. Six of the participants had self-efficacy scores that ranked in top $25 \%$ of the whole survey sample (i.e. high self-efficacy group) whereas the other six participants ranked in the bottom 25\% (i.e. low self-efficacy group). Demographic information of the 12 participants is illustrated in Table 1.

Table 1 Demographic information of the 12 participants

\begin{tabular}{llll}
\hline Participant & Gender & Age (year; month) & Self-efficacy score \\
\hline MH1 & M & $14 ; 1$ & 96 \\
MH2 & M & $13 ; 1$ & 100 \\
MH3 & M & 13 & 100 \\
FH1 & F & $13 ; 6$ & 95 \\
FH2 & F & $13 ; 6$ & 94 \\
FH3 & F & $12 ; 6$ & 100 \\
ML1 & M & $12 ; 6$ & 37 \\
ML2 & M & $13 ; 6$ & 52 \\
ML3 & M & $13 ; 5$ & 43 \\
FL1 & F & $14 ; 9$ & 36 \\
FL2 & F & $12 ; 6$ & 44 \\
FL3 & F & 13 & 66 \\
\hline
\end{tabular}

"M" indicates male; "F" indicates female; " $\mathrm{H}$ " indicates high self-efficacy group; "L" indicates low selfefficacy group. $(N=12)$ 


\section{Materials}

Mathematics Self-Efficacy Scale. The Mathematics Self-efficacy Scale was developed in Chinese by the researcher in accordance with Bandura's (2006) guideline of constructing self-efficacy scales. The scale consists of ten items which represent the mathematics tasks at different difficulty levels based on the authentic status of participants' current mathematics study, for example, "Do calculation as required". Participants were required to rate the extent to which they were certain that they can accomplish each task by choosing a number ranging from "0" (cannot do at all) to "10" (highly certain can do). The overall score of the ten items was regarded as the participant's mathematics self-efficacy level. The internal consistency of the ten items was .95 (Cronbach's $\alpha$ ).

The Statements of Self-Efficacy Sources (Q-Sample). In the Q-sorting procedure, a Qsample refers to a set of prepared statements which reflect the issues under investigation (Watts \& Stenner, 2012). In the present study, the Q-sample was made up of the statements which represent Bandura's (1997) four sources of self-efficacy. The statements were developed by following the guideline of Q-sample development (Watts \& Stenner, 2012). Firstly, an initial set of statements were generated by drawing on the existing measures and interview protocols of the four sources (i.e. Lent et al., 1996b; Usher, 2009; Usher \& Pajares, 2009). These initial statements were then evaluated in a focus group, during which five Chinese students were asked to discuss their understanding of each statement and point out ambiguous or unrepresentative description. Their comments facilitated the refinement of the statements. The final Q-sample was constituted of 18 statements of the four self-efficacy sources (i.e. 4-5 statements for each source, see Table 3 for the statements). Each statement was printed on a paper card (sized $7 \times 10 \mathrm{~cm}$ ) for participants to move around in the Q-sorting procedure.

\section{Procedure}

Face-to-face interviews were conducted with participants individually. Each interview lasted about 30 to $45 \mathrm{~min}$. It started with the participant discussing his/her understanding of "confidence in learning mathematics" with the researcher. Then, the cards with the statements of self-efficacy sources (Q-sample) were shown to the participant one by one in a random order, during which the participant was asked to talk about his/her past experiences elicited by the statement on each card. Participants were encouraged to say as much as possible during this process. This not only enabled the researcher to obtain a large amount of meaningful information, but also helped participants to get familiar with all the statements.

Subsequently, the Q-sorting procedure was carried out. Participants were asked to sort the 18 statements in accordance with the influential power of each statement on their mathematics self-efficacy. All the cards had to be sorted into a response grid with a continuum which ranges from "the least influential" $(-3)$ to "the most influential" $(+3)$ (see Fig. 1). The number of cards for each column was fixed and the distribution of cards was quasi-normal, as has been suggested in the guidelines of Q-sorting (Brown, 1971; Watts \& Stenner, 2012). Participants were obliged to sort the cards to match this 


The least influential
\begin{tabular}{|c|c|c|c|c|c|c|}
\hline \multicolumn{2}{|c|}{-1} & 0 & 1 & \multicolumn{2}{c}{ The most influential } \\
\hline & & & & & & 3 \\
\hline & & & & & & \\
\hline
\end{tabular}

Fig. 1 The quasi-normal distribution of the Q-sorting scale

distribution. In this way, the influence of each source on self-efficacy was differentiated and the relative positions of cards revealed a holistic viewpoint held by the participant about the effects of self-efficacy sources on his/her mathematics self-efficacy.

After participants completed the Q-sorting procedure, a few post-sorting questions were asked to allow participants to elaborate their opinions, for example, "why Card $x$ and Card $y$ were put in the most/least influential column" and "why Card $x$ was more/ less influential than Card y". The interview ended with asking participants to think about if there was any other influential efficacy-relevant information that had not been addressed in the statements. The whole process of interview was video-recorded.

\section{Data Analysis}

The interview data and Q-sorting data were at first analysed separately and then the findings were integrated to address the research questions.

The interview data were analysed in advance of the Q-sorting data in order to minimise the possibility of introducing bias into the qualitative analysis. Two stages of coding were carried out to analyse the interview transcripts. Firstly, content analysis was adopted to investigate the extent to which each participant had been exposed to different sources of self-efficacy in mathematics study. Participants' responses to each statement of self-efficacy sources, that is, their past experiences pertaining to each source were coded into the categories of "never/seldom/sometimes/often/always/ depends". Two researchers coded the whole data separately. Inter-coder reliability was $80.4 \%$ in the first instance. The inconsistent codes were fully discussed before a consensus was reached. The result provides a detailed account of the degree to which each participant had the four self-efficacy sources in their past experiences (see the supplementary material for the result table). Subsequently, the second stage of coding was carried out by adopting the iterative qualitative data analysis method proposed by Miles and Huberman (1994). The analysis started with a list of initial codes which were based on Bandura's (1997) four sources of self-efficacy. The codes were reciprocally refined in the coding process to obtain a better match with the data. This reciprocal process was reiterated until the data and codes met the best fit. The final codes reflected the pattern of interest in the interview data. The codes and corresponding quotes are used to address the research questions of the present study in the following "Findings and Discussion" section.

In this study, the PQmethod software (Schmolck, 2014) was used to input and analyse the Q-sorting data. By-person factor analysis was conducted to account for the variance of the Q-sorting data. Unlike conventional factor analysis, by-person factor analysis correlates participants instead of items. The participants who produced similar Q-sorting outcomes (i.e. shared a similar viewpoint) were identified as a certain "participant group" and the configuration of their Q-sorting outcomes emerged as a 
latent "factor" which demonstrated the shared viewpoint (Watts \& Stenner, 2012). Rather than based on hypotheses that individual differences result from demographic or personality traits alike, by-person factor analysis takes a bottom-up approach to reveal consensus and disagreement among participants' viewpoints based on the variance of the data per se. As a result, researcher-imposed bias can be minimised in the analysis. In this study, principal component analysis with Varimax rotation was conducted with the Q-sorting data. The result was integrated with the findings of qualitative analysis to address the research questions.

\section{Findings and Discussion}

Corresponding to the two research questions, the discussion section firstly demonstrates how students of different genders and mathematics self-efficacy levels differed in their past experiences of the sources of mathematics self-efficacy. Subsequently, the different viewpoints of the effects of each source on mathematics self-efficacy are presented. Finally, three aspects of the cultural variation identified in this study are discussed to set the stage for future research.

\section{Different Degrees of Exposure to Sources of Self-Efficacy}

Mastery Experience. The interview data show that all participants with high self-efficacy reported abundant mastery experiences whereas their counterparts with low self-efficacy recalled constant setbacks in learning mathematics. Low self-efficacy participants claimed that they had got used to repeated failures and described their occasional achievement as "a big surprise" (e.g. ML1, ML3, FL1, FL2). In contrast, high self-efficacy participants indicated that even though they had occasional failures which were deemed as a threat to self-efficacy, they would still hold a belief that they could do well next time by working harder (e.g. MH3: “...I can keep working hard for next time. Just like at the beginning of Grade 6, I didn't learn maths very well, but I kept working very hard, then my scores improved"). Usher (2009) also reported a similar finding that high self-efficacy students not only had more mastery experiences but also had a more positive attitude towards failures, in comparison with low self-efficacy students.

Vicarious Experience. The interview data demonstrate substantial disparities between high and low self-efficacy participants with regard to vicarious experience. All six high self-efficacy participants described their experiences of actively seeking competition and highlighted the benefits of social comparison (e.g. FH2: "I think it is necessary to compete with each other when learning mathematics. Because when I find someone who is doing better than me, I'll work much harder to catch up. If others are not as good as I am, I always tell myself that I would not let them surpass me easily. I think competing with classmates motivates learning"). In contrast, five of the six low selfefficacy participants (except FL3) admitted that they tended to avoid social comparison in mathematics study because comparison often generated frustration and a sense of loser (e.g. FL1: "It's impossible for me to compete with others. You can compete with me in term of English or literature, but not mathematics, because my mathematics is crap"; ML2: "Comparison made me a loser"). 
Meanwhile, the attitudes towards peer modelling were slightly different between high and low self-efficacy participants. While low self-efficacy participants paid more attention to the instructive function of peer models (e.g. FL2: "I want to learn from models, do what they do."), high self-efficacy participants seemed to lay more emphasis on the comparative function of peer models (e.g. FH2: "Watching others do well in mathematics motivates me to work harder, because I really want to catch up with others, to be as good and even do better than them"). This corresponds to Chan and Lam's (2008) finding that students' eagerness to surpass peer models may mask the instructive value of modelling.

Social Persuasion. In this study, girls recalled receiving more social persuasion than boys, especially from parents. All six girls claimed that their parents often gave them evaluative feedback about their mathematics learning (e.g. FH1: "It often happens. For example, if I got a good score in mathematics exams, they [parents] always praise me"). In contrast, four of the six boys indicated that their parents seldom commented on their mathematics learning (e.g. MH1: "It's just, no praise, no criticism"). Similar findings of girls receiving more social persuasion in mathematics study were also reported in previous studies (e.g. Lent et al., 1996b).

The difference between high and low self-efficacy participants is also noticeable. All six low self-efficacy participants indicated that they received more negative feedback than praise. Therefore, they valued those rare compliments and described such experiences as having a boost in their self-efficacy, albeit sometimes short-lasting (ML1: "The teacher praised me once. I felt really happy and confident. But then my score turned out to be bad, again my confidence dropped"). This finding concurs with Bandura's (1997) theory that social persuasion has limited influence on self-efficacy without the complements of other sources, in particular, mastery experiences. On the other hand, high self-efficacy participants seemed to hold a more positive attitude towards feedback, even when receiving occasional negative feedback (e.g. FH3: "When the teacher criticizes me, I think it means that there is still space for improvement. It pushes me to work harder"). Usher (2009) also found such resilience against negative feedback among high self-efficacy students in her interview study.

Physiological and Affective States. The present study has touched upon a wide range of physiological and affective states during mathematics study. The participants expressed a variety of arousals, ranging from excitement, pride, delight, satisfaction and superiority to anxiety, agitation, fatigue, boredom, envy, depression, sleepiness, frustration and anger. This enriches the findings of previous research which mainly focused on anxiety (Usher \& Pajares, 2009).

The data show that girls reported experiencing more anxiety than boys in mathematics learning, especially when confronted with difficult tasks. For example, FL2 described her overwhelming anxiety by saying that "Sometimes during maths examination... I'm so nervous, nervous to death. Sometimes the teacher asks me a question and I can't answer it, I'm nervous to death". On the contrary, boys seldom mentioned anxious arousals of studying mathematics in the interviews and even those with low self-efficacy described themselves as "quite calm" (ML1, ML3). Similar findings have been reported in some previous studies (Britner \& Pajares, 2006; Joët et al., 2011; Lent et al., 1996b). Yet, boys mentioned feeling irritated/fretful much more often than girls in the interviews (e.g. MH2: "sometimes I feel a little bit less confident because I 
become irritated"; ML1: "if the homework is difficult, I become fretful"). On the other hand, girls recalled more times in the interviews that mathematics activities cost them large amount of energy (e.g. FL1: "I spend almost all my time on mathematics homework. I am just so exhausted afterwards"). Future research may explore these subtle gender differences in detail.

In terms of the difference between high and low self-efficacy participants, the interview data show that high self-efficacy participants experienced more feelings of excitement, pride, delight, satisfaction and superiority during mathematics activities than their low self-efficacy counterparts who reported more boredom and frustration. It seems that good performances brought about a sense of fulfilment, which in turn motivated students to put forth more effort to achieve better performances, thereby generating a self-empowering circle, vice versa. This finding highlights the role of effect in motivating learning.

High and low self-efficacy participants also differed in their attitudes towards emotion management in mathematics learning. As a matter of fact, high self-efficacy participants also reported sometimes experiencing negative arousals, particularly when confronted with obstacles and setbacks. They acknowledged the detrimental effects of these arousals and stressed the importance of using effective strategies to manage these emotions (e.g. MH1: "You can't study well in bad mood. I will adjust myself so that I can concentrate on learning"; FH3: "I would talk to myself, tell myself to calm down"). In contrast, low self-efficacy participants seemed to be rather passive in the face of negative arousals (e.g. ML1: "[when feeling frustrated because of mathematics study], I don't know what to do. There's nothing I can do. I guess it just goes away the next day"). Therefore, it is not that high self-efficacy participants seldom underwent any negative arousals, but rather they were more capable of emotional management than low self-efficacy peers. Likewise, Usher (2009) also reported a difference between high and low self-efficacy students in employing strategies of emotional management. These findings suggest that previous survey research, which normally correlated self-efficacy with negative arousals (sometimes solely anxiety), may have oversimplified the complicated mechanisms underlying students' interpretation of their physiological and affective arousals. Future studies can benefit from a qualitative or mixed-method approach, which will generate more thorough findings.

\section{Different Viewpoints of the Influence of Self-Efficacy Sources}

The Q-sorting procedure was used to investigate participants' viewpoints of the influence of self-efficacy sources. According to the result of by-person factor analysis, two factors emerged from the Q-sorting data. Factor 1 (eigenvalue $=3.16$ ) explained $27 \%$ of the overall variance of the data and factor 2 (eigenvalue $=2.02$ ) accounted for $16 \%$ of the variance. Table 2 shows the factor loadings of the 12 Q-sorting outcomes (i.e. Q-sorts) which were carried out by the participants. The result shows that participants $\mathrm{MH} 1, \mathrm{MH} 3, \mathrm{FH} 1, \mathrm{FH} 2^{1}$ and $\mathrm{FH} 3^{1}$ were significantly loaded on factor 1 , which

\footnotetext{
${ }^{1}$ It is noted that FH2 and FH3 also have a loading of 0.5472 and -0.5756 on factor 2, respectively. But these loadings are not significant at the 0.01 level (Brown, 1971). Considering their significant loadings on factor 1 (0.7531 and 0.7751$)$, it is reasonable to adopt this factor solution and retain FH2 and FH3 in factor 1(Watts \& Stenner, 2012).
} 
Table 2 The rotated loadings of each Q-sort on the two factors

\begin{tabular}{lcr}
\hline Q-sort & Factor 1 & Factor 2 \\
\hline MH1 & $0.7391^{*}$ & 0.1408 \\
MH2 & 0.2567 & -0.0270 \\
MH3 & $0.6499^{*}$ & -0.1147 \\
FH1 & $0.7520^{*}$ & -0.1734 \\
FH2 & $0.7531^{*}$ & 0.5472 \\
FH3 & $0.7751^{*}$ & -0.5756 \\
ML1 & 0.0596 & $0.7743^{*}$ \\
ML2 & $-0.6477^{*}$ & 0.1473 \\
ML3 & 0.1859 & -0.3191 \\
FL1 & 0.0746 & $0.6116^{*}$ \\
FL2 & 0.2372 & 0.0864 \\
FL3 & -0.0284 & 0.3050 \\
Eigenvalue & 3.16 & 2.02 \\
Variance $(\%)$ & 27 & 16 \\
\hline
\end{tabular}

“*” indicates the factor loading is significant $(p<.01)$

indicates that they held a similar viewpoint. Conversely, participant ML2 had a negatively significant loading on factor 1, which suggests that ML2 held an opposite viewpoint to that of the aforementioned five participants. As for factor 2, participants ML1 and FL1 were significantly loaded on it, which suggests that they shared a similar viewpoint which was considerably different from factor 1. Participants MH2, ML3, FL2 and FL3 were not significantly loaded on either factor, which indicates that their viewpoints were relatively idiosyncratic in the present sample.

Table 3 illustrates the representative item scores of the two emergent factors, each of which represents a shared viewpoint about the influences of self-efficacy sources. The representative item scores are obtained through the weighted averaging procedure. The item scores of each participant who is significantly loaded on the factor are multiplied by the corresponding factor loadings to obtain the weighted average score which are then arranged in order and subsequently transformed into the scores used in the original grid to facilitate the interpretation of item scores. As a result, the factor item scores demonstrate the best-fitting representation of the shared viewpoint. In order to understand the two shared viewpoints, Q-sorting outcomes were synthesised with the interview data to demonstrate how participants perceived the influence of each source on their mathematics self-efficacy.

Factor 1: the Shared Viewpoint of Five High Self-Efficacy Students. Five high selfefficacy participants (except MH2) were significantly loaded on factor 1 . The result of item scores showed that they regarded competition in mathematics study as the most influential information (Card18: 3). Evidence from the interview data showed that high self-efficacy participants actively sought competition and tended to judge their abilities based on comparison (e.g. FH2: "I think it is necessary to compete with each other when learning mathematics"; FH3: "The ranking of my score in the class determines 
Table 3 The item scores of the two factors

Factors

Statements

F1 F2

1. I get a satisfactory score in mathematics examination.

22

2. I solve the mathematics problems which my classmates cannot work out.

11

3. I finish my mathematics homework every day and get good marks.

$-31$

4. I correctly answer the teacher's question in mathematics class.

5. Although I have made great effort, I still do not get a good score in mathematics examination. $1 \quad-1$

6. Watching my classmates do better in mathematics encourages me to learn it better. $\quad 2 \quad 3$

7. When the teacher shows us how to solve one mathematics problem, I think I will also solve $\quad-10$ similar questions by myself.

8. When I find other students using better learning strategy, I think I will learn mathematics better $0 \quad 3$ using the same strategy.

9. My father or my mother is good at mathematics. $\quad-30$

10. The mathematics teacher gives me evaluative feedback about my mathematics study. $\quad \begin{array}{ll}0 & 2\end{array}$

11. My parents give me evaluative feedback about my mathematics study. $\quad-1 \quad 1$

12. My classmates tell me that I am good at mathematics. $\quad-2-2$

13. Other people tell me that I have good ability to learn mathematics well. $-2-3$

14. Learning mathematics makes me nervous and agitated. $\quad 1 \quad-3$

15. Working out mathematics problems takes a lot of energy. $\quad 0 \quad-2$

16. My mind becomes absent during mathematics class. $\quad 0 \quad 0$

17. Mathematics makes me feel depressed and frustrated. $\quad 3-1$

18. I compete with other students when learning mathematics. $\quad 3 \quad 0$

$"-3 "=$ "the least influential"; "0" = "moderately influential"; "3" = "the most influential"

how confident I am"). According to them, success meant "surpassing others in examination" (MH1, FH1, FH3), "getting the highest score" (FH2) or "always being the top three" (MH3). They recalled their self-efficacy being considerably strengthened when defeating their competitors. In contrast, being surpassed by others would shake their self-efficacy and generate frustration. Thus, they recognised the great impact of social comparison on their self-efficacy (e.g. MH3: "outperforming my competitors in mathematics can improve my confidence to the largest extent"). Without surprise, the statements that implied social comparison were also ranked as relatively influential (Card6: 2; Card2: 1).

In addition to social comparison, the five high self-efficacy participants also stressed the considerable effect of negative emotions on their self-efficacy (Card17: 3; Card14: 1). They regarded negative emotions evoked by mathematics as a serious issue, even though they recalled experiencing more positive feelings (e.g. excitement, pride, delight, satisfaction and superiority) than negative feelings (e.g. irritableness, fatigue, frustration and agitation) when studying mathematics. Since they were not used to having negative arousals due to mathematics study, they were very sensitive to these emotions and paid great attention to any detrimental effects of these arousals on their performances as well as self-efficacy beliefs (e.g. FH1: "When I'm in bad mood, I become absent-minded. And then I always feel agitated. Anyway, if I'm in bad mood, I 
cannot learn well"). They underscored the importance of using effective strategies to manage these negative emotions (e.g. MH1: "I need to calm down and concentrate on the work. I tell myself and I take a deep breath"). Both the Q-sorting and the interview data show evidence to support the considerable impact of negative emotions on the five high self-efficacy participants.

Another two statements which were ranked as relatively influential were regarding mastery experiences, in particular, examination outcomes (Card1: 2; Card5: 1). Not all mastery experiences were deemed as influential by the five high self-efficacy participants. The statements regarding correctly answering questions in mathematics class (Card4: - 1) and finishing homework (Card3: -3) were ranked as being "less influential" and "the least influential", respectively. The participants explained that it was because the two tasks indicated on cards 3 and 4 were too easy and too predictable for them. In contrast, mathematics examinations could be challenging and therefore had stronger influence on their self-efficacy.

The statements tapping vicarious learning were ranked in the range from "the least influential" to "moderately influential". More specifically, peer modelling (Card8: 0) was more influential than teacher modelling (Card: -1 ), which was more influential than parent modelling (Card 9: - 3). The participants pointed out that it was because (a) they shared more similarities with peers and (b) peer modelling generated social comparison which always affected their self-efficacy (e.g. MH3: "When I find that other students are doing well, it motivates me to do better. I need to be better"). This finding is consistent with Bandura's (1997) postulation that the more similar the model is to the observer, the more influential it is likely to be.

In terms of social persuasion, these high self-efficacy participants regarded appraisals from teachers as moderately influential (Card10: 0) whereas appraisals from parents, peers and others were ranked as being "less influential" (Card11: - 1, Card12/13: - 2). According to the participants, teachers' appraisals carried more weight because the participants regarded teachers as authorities. Nonetheless, social persuasion seemed to have limited influence on self-efficacy of these participants who explained that it was because they had got accustomed to compliments about their mathematics studies. It was the occasional criticism that got the attention of these high self-efficacy participants. They demonstrated a positive attitude towards criticism, acknowledging the benefits of necessary criticism to their mathematics study (e.g. MH1: "Sometimes I am too confident, like cocky, I become careless and don't do work properly. My teachers' or parents' criticism makes me realise this. It is helpful"). Based on these findings, it seems that these high self-efficacy participants had relatively robust self-efficacy beliefs which were not easily shaken by social persuasion (e.g. FH1: "I know my ability. It's unlikely that I will change it simply because someone says something"). This is at odds with some previous studies which suggested that students from collectivistic cultural backgrounds tended to be susceptible to social persuasion (Ahn et al., 2016b; Kitayama \& Markus, 2014). Actually, the current findings reveal within-culture variations concerning this issue. Instead of regarding Chinese students as an analogous sample, this study endeavours to shed light on individual difference in students' perceptions of self-efficacy sources, thereby extending the findings of previous studies.

Factor 2: the Shared Viewpoint of Two Low Self-Efficacy Students. Factor 2 represented the shared viewpoint of ML1 and FL1. Although only two participants significantly 
loaded on this factor, the result of factor analysis shows that factor 2 has an eigenvalue of 2.02 and accounts for $16 \%$ of the variance of Q-sorting data. According to the Kaiser-Guttman criterion (Guttman, 1954; Kaiser, 1960), it is sensible to retain this factor. As this factor represents the shared viewpoint of two low self-efficacy participants, it contrasts with the shared viewpoint of high self-efficacy participants (i.e. factor 1 ), which may of interest to readers.

It is noticeable that both participants accentuated the strong influence of peer modelling on their self-efficacy (Card6/8: 3). They focused on the instructive function of peer modelling, rather than fixating on its comparative function. ML1 mentioned many times his eagerness to follow peer models so that he could learn mathematics "as well as others". FL1 described that "when I see my classmates complete the homework and hand it to the teacher, I think that I should also do my homework. So I ask them how to do it, something like that. Then I can finish my homework. If other students do not do the homework, I won't do it because I won't know how to do it". Apparently, peer modelling played a vital role in promoting self-efficacy for both participants. However, teacher and parent modelling were regarded as moderately influential (Card7/9: 0). This again supports Bandura's (1997) postulation that modelling has stronger effects on self-efficacy when there are more similarities between the model and the observer.

In terms of social comparison, both participants claimed that they never initiated competition in mathematics study with other students (e.g. ML1: "I never want to compete with anyone in mathematics."). Yet, they still ranked competition as having moderate influence on their self-efficacy (Card18: 0). Despite avoiding competition, both participants admitted that it was inevitable to be affected by social comparison in mathematics study. This is in line with previous findings about the prevalence of competition in Chinese educational environments (King, McInerney, \& Watkins, 2012).

As for social persuasion, evaluative feedback from teachers and parents was ranked as being relatively influential (Card10: 2, Card11: 1). Both participants indicated that because they rarely received compliments from their teachers or parents, they cherished the experience of being praised by adults. They both mentioned experiencing temporary boost in self-efficacy because of praise (e.g. FL1: "The teacher praised me once. Then the confidence, my confidence just boosted"). Unlike their high self-efficacy counterparts who always received abundant compliments, the two low self-efficacy participants fully acknowledged the considerable effects of encouraging messages from teachers and parents on their self-efficacy. In contrast, compliments from peers and others were considered being less convincing, thereby less influential to self-efficacy (Card12: - 2, Card13: - 3). This finding supports Ahn, Bong, and Kim's (2016a) suggestion that the influence of social persuasion on self-efficacy should be differentiated in terms of the corresponding social models.

With respect to mastery experience, the statement about getting a satisfactory examination score was ranked as being relatively influential (Card1: 2). However, the statement about failing in examination despite great effort was ranked as being less influential (Card5: - 1). The participants explained that it was because they seldom got satisfactory examination scores no matter how hard they tried (e.g. ML1: "I get used to it"; FL1: "This is for sure. It doesn't matter. I just can't do well in mathematics examination"). Therefore, a good examination score was regarded as an unusual 
booster for self-efficacy whereas failure in examination was seen as expected and hence less influential to their self-efficacy. Meanwhile, the mastery experiences of solving mathematics problems and completing homework were ranked as relatively influential (Card2/3: 1). The two participants indicated that the accumulating effects of small mastery experiences could be as powerful as success in examinations. Apparently, they had very different perceptions of mastery experiences from the aforementioned five high self-efficacy participants.

Moreover, the two participants regarded negative physiological and affective states as being either the least influential (Card14: -3 ) or less influential (Card 15: -2 , Card17: -1 ), despite acknowledging that they frequently had negative emotions when engaged in mathematics study. They further explained that it was the fact that they often felt depressed, agitated and anxious due to mathematics that made them see these negative states as common for learning mathematics (e.g. FL1: "It's no big deal, I have got used to them."), which resulted in the relatively weak effects of negative emotions on their mathematics self-efficacy. This finding again poses a striking contrast to that of the five high self-efficacy participants in factor 1 .

Idiosyncratic Viewpoints. As mentioned earlier, five participants were not clustered into groups, which mean that they had rather diverse viewpoints of the influence of selfefficacy sources. It is striking to find out that students, especially those with low selfefficacy, perceived the sources of mathematic self-efficacy in diverse ways. This diversity was not underscored in previous studies taking a quantitative approach. While generalisation is important in educational research, it is also meaningful to draw attention to individual differences and raise awareness of the existing diversity. Readers who are interested in the diverse viewpoints of these participants can find detailed analysis in the supplementary materials of this paper.

\section{Cultural Variation in the Sources of Mathematics Self-Efficacy}

While most of the studies of self-efficacy sources were conducted in western contexts (Usher \& Pajares, 2008), the present study focuses on students with a collectivistic cultural background, which provides an opportunity to explore cultural variation in this issue. Based on the findings, cultural variation seems to be manifested in participants' perceptions of the following three aspects.

Firstly, effort was highly valued by all the participants, including $\mathrm{MH} 2$ who claimed that he could achieve good performance with little effort as well as the participants with low self-efficacy who admitted that it was not uncommon for them to get bad examination scores after putting forth great effort. All the participants shared a common belief that the more effort one exerts, the better one can become at mathematics. Just as FH1 said, "If you don't put forth efforts, you cannot be confident. I think it is true for all the subjects that you cannot make great academic achievement unless you work hard". While Bandura (1997) suggested that students may read great effort expenditure as a sign of ability deficiency, the present participants seemed to lay more emphasis on the potential improvement in competence which resulted from effort expenditure. As a result, effort was related to enhancement in self-efficacy in the context of the present study. Participants of the present study hardly saw expanding great effort as a sign of 
deficiency in competence but rather as one of self-regulatory skills which led to improvement in competence. Participants' attitudes towards effort reflected the ethos of learning in Chinese schools: diligence is highly valued (Li, 2003) and the belief that effort leads to ability improvement is instilled and reinforced in students' mind (Bergen \& Mi, 1995). This may partially explain why the present finding was at odds with Bandura's (1997) assumption based on western learning ethos. Moreover, this issue relates to Dweck's (2006) theories of intelligence, which suggests that some people believe ability is a fixed trait (i.e. fixed mindset) whereas some people believe ability can be cultivated and improved (i.e. growth mindset). Very few studies have examined Chinese students' theories of intelligence (Chen \& Wong, 2015), let alone the relationship between self-efficacy and different mindsets in Chinese educational contexts. Yet, it is a topic worth exploring and future studies may seek to shed some light on this issue.

Secondly, participants mentioned "face" issue many times in interviews. As an indigenous concept in Chinese context, "face" issue refers to the need to "save face" (i.e. sustain dignity and self-esteem) and to avoid "losing face" (i.e. embarrassment and shame) in public (Cheng, 1986; Wu, 2004). "Face" issue implies some subtle information that is culturally specific (Wu, 2004). Participants indicated that "face" issue amplified the effects of various self-efficacy sources. For example, MH2, FH1 and FL1 pointed out that being publicly praised in front of their classmates had much stronger effects on self-efficacy than compliments given in privacy because the former enabled them to "gain face". MH1 and FH3 recalled their experiences of publically claiming their success in mathematics examinations in order to feel "gaining face". MH2 and MH3 stressed their needs to "fight for face" in competition, stating that "if I lose in competition, I'll feel that I'm losing face" (MH2) and "losing face makes me depressed all day. I don't want to do mathematics at all" (MH3). It seems that the feeling of "losing face" would evoke negative emotions and debilitate motivation in learning. Actually, "face" issue can be related to adolescents' need of maintaining self-esteem, which is not particular for Chinese students but rather universal among adolescents of different cultural backgrounds (Mruk, 2013; Wu, 2004), even though the influence of "face" issue may be more a manifest in Chinese cultural background. While there have been ample studies of the relationship between self-esteem and self-efficacy (e.g. Judge, Erez, Bono, \& Thoresen, 2002; Lane, Lane, \& Kyprianou, 2004), it is of interest to explore why "face" issue has such strong impact on Chinese students' mathematics self-efficacy in future studies.

Finally, the findings mentioned earlier showed that participants with high selfefficacy tended to actively initiate competition whereas almost all the participants with low self-efficacy seemed to intentionally avoid competition. Several previous studies suggested that students in collectivistic cultures were relatively sensitive to social comparison in general, for example, Klassen (2004) found that South Asian (Indo Canadian) immigrant students were more strongly influenced by social comparison than Anglo Canadian students. Similarly, Chan and Lam (2008) found that in a competitive context, Hong Kong students tended to regard models with superior performance as a threat to them and therefore had lower self-efficacy beliefs. In the present study, students with high or low mathematics self-efficacy expressed divergent attitudes towards competition. In contrast to some previous studies which suggested that competition tended to remain at a low level in classrooms of collectivistic cultures 
(Markus \& Kitayama, 1991; Triandis, 2001), the participants with high self-efficacy in the present study were actively involved in a high level of competition in mathematics. However, the participants with low self-efficacy regarded competition as detrimental to their self-efficacy and sought to avoid competition in mathematics study. These findings suggest that competition can be a two-edged sword which has a different impact on self-efficacy under different circumstances. In the same vein, some previous studies in western contexts also acknowledged both positive and negative effects of competition on achievement motivation of students (e.g. Cantador \& Conde, 2010). Future studies may seek to further explore the potential cultural variation in the effects of competition on students' self-efficacy development.

\section{Conclusions}

By incorporating semi-structured interview with the Q-sorting procedure, the present study conducted an in-depth investigation into the sources of mathematics self-efficacy of Chinese junior high school students. Findings show that students not only had different experiences of the four sources but also held diverse viewpoints about the effects of each source on their mathematics self-efficacy. Even for a single source, participants differentiated the effects of different events pertaining to the source. Therefore, it is arbitrary to reach the conclusion that a certain source is always more influential than the others. While previous quantitative studies mainly focused on the degree to which each of the four sources was associated with self-efficacy, the present study demonstrates more in-depth information about students' perceptions of the sources, thereby revealing the complexity in self-efficacy development.

In this study, girls recalled receiving more social persuasion and experiencing more anxiety than boys in mathematics learning, which is consistent with several previous studies (e.g. Britner \& Pajares, 2006; Lent et al., 1996b). However, according to the result of by-person factor analysis, girls' perceptions of the influence of each source were not significantly different from boys'. This result has expanded the existing findings of gender difference in the development of mathematics self-efficacy. Future studies can further explore the potential gender difference in self-efficacy of other STEM subjects.

On the other hand, the difference between high and low self-efficacy participants is noticeable. Five of the high self-efficacy participants shared a similar viewpoint about the influence of self-efficacy sources whereas the low self-efficacy participants seemed to hold more diverse viewpoints, four of whom expressed relatively idiosyncratic viewpoints about the influence of each source on their self-efficacy. The findings highlight individual differences in this issue, suggesting that there is no one-size-fitsall approach to promoting students' mathematics self-efficacy. Teachers should draw attention to contextual and individual factors when fostering development of mathematics self-efficacy in students.

Furthermore, by focusing on Chinese students' self-efficacy development, this study reveals both similarities and divergences of opinions between Chinese students and their western counterparts. The cultural variation in the effects of efforts, competition and face issue on self-efficacy development has been thoroughly discussed to underline the importance of taking contextual factors into consideration in the research of self- 
efficacy sources. More studies are needed to shed light on self-efficacy development of students from non-western cultural backgrounds.

Finally, this empirical investigation took advantage of the combination of semistructured interview and Q-sorting procedure which has demonstrated multiple methodological advantages in unveiling the complexity in self-efficacy development. Yet, it is important to point out the limitations of this study to stimulate future refinement. In this study, considering the length of interview and students' attention time, a compromise was made to restrain the number of statements involved in the Q-sorting. It is undeniable that to include more statements about the sources of self-efficacy in the Qsorting may generate a more comprehensive understanding of how students build up and shape their mathematics self-efficacy. Future studies can refine the statements based on this study, thereby benefiting more from the Q-sorting. Also, this study made a comparison between students of high and low mathematics self-efficacy but did not involve students of middle level of self-efficacy in the research. It is likely that students of middle level of self-efficacy may differ from students of high and low self-efficacy in terms of their experiences and interpretations of the sources. Therefore, future studies should consider including students of various levels of self-efficacy in the Q-sorting activity in order to generate more comprehensive representation of students' perceptions of self-efficacy sources. Despite the limitations, this study has generated interesting findings of how Chinese junior high school students perceived the sources of selfefficacy in mathematics learning, which contributes to future research on the development of self-efficacy.

Acknowledgements I would like to thank Dr. Ros McLellan and Dr. Julia Flutter from the Faculty of Education, University of Cambridge for their guidance and support with the project.

Open Access This article is distributed under the terms of the Creative Commons Attribution 4.0 International License (http://creativecommons.org/licenses/by/4.0/), which permits unrestricted use, distribution, and reproduction in any medium, provided you give appropriate credit to the original author(s) and the source, provide a link to the Creative Commons license, and indicate if changes were made.

\section{References}

Ahn, H. S., Bong, M., \& Kim, S. (2016a). Social models in the cognitive appraisal of self-efficacy information. Contemporary Educational Psychology, 48, 149-166.

Ahn, H. S., Usher, E. L., Butz, A., \& Bong, M. (2016b). Cultural differences in the understanding of modelling and feedback as sources of self-efficacy information. British Journal of Educational Psychology, 86(1), 112-136.

Bandura, A. (1997). Self-efficacy: The exercise of control. New York, NY: W H Freeman/Times Books/ Henry Holt \& Co.

Bandura, A. (2006). Guide for constructing self-efficacy scales. Self-Efficacy Beliefs of Adolescents, 5, 307337.

Bergen, T. J., \& Mi, H. (1995). An analysis and review of Confucian philosophy as the basis for Chinese education. International Education, 24(2), 40-52.

Britner, S. L., \& Pajares, F. (2006). Sources of science self-efficacy beliefs of middle school students. Journal of Research in Science Teaching, 43(5), 485-499.

Brown, S. R. (1971). The forced-free distinction in Q technique. Journal of Educational Measurement, 8(4), 283-287. 
Burnham, J. R. (2011). A case study of Mathematics self-efficacy in a freshman engineering mathematics course(Unpublished master's thesis). Washington State University, USA.

Butz, A. R., \& Usher, E. L. (2015). Salient sources of early adolescents' self-efficacy in two domains. Contemporary Educational Psychology, 42, 49-61.

Cantador, I., \& Conde, M. (2010). Effects of competition in education: A case study in an e-learning environment. In M. B. Nunes \& M. McPherson (Eds.), The proceedings of IADIS international conference e-learning 2010 (pp. 11-18). Freiburg, Germany.

Caprara, G. V., Fida, R., Vecchione, M., Del Bove, G., Vecchio, G. M., Barbaranelli, C., \& Bandura, A. (2008). Longitudinal analysis of the role of perceived self-efficacy for self-regulated learning in academic continuance and achievement. Journal of Educational Psychology, 100(3), 525-534.

Chan, J. C., \& Lam, S. (2008). Effects of competition on students' self-efficacy in vicarious learning. British Journal of Educational Psychology, 78(1), 95-108.

Chen, W., \& Wong, L. (2015). Chinese mindset: Theories of intelligence, goal orientation and academic achievement in Hong Kong students. Educational Psychology, 35(6), 714-725.

Cheng, C. Y. (1986). The concept of face and its Confucian roots. Journal of Chinese Philosophy, 13(3), 329348.

Dweck, C. S. (2006). Mindset: The new psychology of success. New York, NY: Random House.

Guttman, L. (1954). Some necessary conditions for common factor analysis. Psychometrika, 19, 149-161.

Joët, G., Usher, L., \& Bressoux, P. (2011). Sources of self-efficacy: An investigation of elementary school students in France. Journal of Educational Psychology, 103(3), 649-663.

Judge, T. A., Erez, A., Bono, J. E., \& Thoresen, C. J. (2002). Are measures of self-esteem, neuroticism, locus of control, and generalized self-efficacy indicators of a common core construct? Journal of Personality and Social Psychology, 83(3), 639-710.

Kaiser, H. F. (1960). The application of electronic computers to factor analysis. Educational and Psychological Measurement, 20, 141-151.

King, R. B., McInerney, D. M., \& Watkins, D. A. (2012). Competitiveness is not that bad... at least in the East: Testing the hierarchical model of achievement motivation in the Asian setting. International Journal of Intercultural Relations, 36(3), 446-457.

Kiran, D., \& Sungur, S. (2012). Middle school students' science self-efficacy and its sources: Examination of gender difference. Journal of Science Education and Technology, 21(5), 619-630.

Kitayama, S., \& Markus, H. R. (2014). Culture and the self: Implications for cognition, emotion, and motivation. In P. G. Altbach, K. Arnold, \& I. C. King (Eds.), College student development and academic life: psychological, intellectual, social and moral issues (pp. 264-293). New York, NY: Garland Publishing, Inc.

Klassen, R. M. (2004). A cross-cultural investigation of the efficacy beliefs of south Asian immigrant and Anglo Canadian nonimmigrant early adolescents. Journal of Educational Psychology, 96(4), 731-742.

Lane, J., Lane, A. M., \& Kyprianou, A. (2004). Self-efficacy, self-esteem and their impact on academic performance. Social Behavior and Personality: An International Journal, 32(3), 247-256.

Lent, R. W., Brown, S. D., Gover, M. R., \& Nijjer, S. K. (1996a). Cognitive assessment of the sources of mathematics self-efficacy: A thought-listing analysis. Journal of Career Assessment, 4(1), 33-46.

Lent, R. W., Lopez, F. G., Brown, S. D., \& Gore, P. A. (1996b). Latent structure of the sources of mathematics self-efficacy. Journal of Vocational Behavior, 49(3), 292-308.

Li, J. (2003). US and Chinese cultural beliefs about learning. Journal of Educational Psychology, 95(2), 258267.

Lopez, F. G., \& Lent, R. W. (1992). Sources of mathematics self-efficacy in high school students. The Career Development Quarterly, 41(1), 3-12.

Markus, H. R., \& Kitayama, S. (1991). Culture and the self: Implications for cognition, emotion, and motivation. Psychological Review, 98(2), 224-253.

Miles, B., \& Huberman, M. (1994). Qualitative data analysis: An expanded sourcebook. Thousand Oaks, CA: Sage.

Mruk, J. (2013). Self-esteem and positive psychology: Research, theory, and practice. New York, NY: Springer Publishing Co, Inc.

Oettingen, G. (1995). Cross-cultural perspectives on self-efficacy. In A. Bandura (Ed.), Self-efficacy in changing societies (pp. 149-176). New York, NY: Cambridge University Press.

Phan, H. P. (2011). Interrelations between self-efficacy and learning approaches: A developmental approach. Educational Psychology, 31(2), 225-246.

Prat-Sala, M., \& Redford, P. (2012). Writing essays: Does self-efficacy matter? The relationship between selfefficacy in reading and in writing and undergraduate students' performance in essay writing. Educational Psychology, 32(1), 9-20. 
Schmolck, P. (2014). PQ method manual. Retrieved from http://schmolck.userweb.mwn. de/qmethod/pqmanual.htm.

Stephenson, W. (1953). The study of behavior: Q-technique and its methodology. Chicago, IL: University of Chicago Press.

Triandis, H. C. (2001). Individualism-collectivism and personality. Journal of Personality, 69(6), 907-924.

Usher, E. L. (2009). Sources of middle school students' self-efficacy in mathematics: A qualitative investigation. American Educational Research Journal, 46(1), 275-314.

Usher, E. L., \& Pajares, F. (2006a). Inviting confidence in school: Invitations as a critical source of the academic self-efficacy beliefs of entering middle school students. Journal of Invitational Theory and Practice, 12, 7-16.

Usher, E. L., \& Pajares, F. (2006b). Sources of academic and self-regulatory efficacy beliefs of entering middle school students. Contemporary Educational Psychology, 31(2), 125-141.

Usher, E. L., \& Pajares, F. (2008). Sources of self-efficacy in school: Critical review of the literature and future directions. Review of Educational Research, 78(4), 751-796.

Usher, E. L., \& Pajares, F. (2009). Sources of self-efficacy in mathematics: A validation study. Contemporary Educational Psychology, 34(1), 89-101.

Watts, S., \& Stenner, P. (2012). Doing Q methodological research: Theory, method \& interpretation. London, England: Sage.

Wu, T. (2004). A review on the study of the concept of Mianzi and its function. Psychological Science, 27(4), 927-930.

Zeldin, A. L., \& Pajares, F. (2000). Against the odds: Self-efficacy beliefs of women in mathematical, scientific, and technological careers. American Educational Research Journal, 37(1), 215-246.

Zeldin, A. L., Britner, S. L., \& Pajares, F. (2008). A comparative study of the self-efficacy beliefs of successful men and women in mathematics, science, and technology careers. Journal of Research in Science Teaching, 45(9), 1036-1058.

Zimmerman, B. J. (2000). Self-efficacy: An essential motive to learn. Contemporary Educational Psychology, $25(1), 82-91$. 